\title{
ESPECTROS DE CONTROL PARA EL VALLE DE LOS CHILLOS
}

\author{
CONTROL SPECTRA FOR CHILLOS VALLEY \\ Roberto Aguiar ${ }^{(1)}$, Diego Quizanga ${ }^{(2)}$ y Andrea Chicaiza ${ }^{(3)}$ \\ (1) Departamento de Ciencias de la Tierra y la Construcción \\ Universidad de Fuerzas Armadas ESPE \\ Av. Gral. Rumiñahui s/n. Valle de los Chillos, Ecuador \\ rraguiar@espe.edu.ec \\ (2) Facultad de Ingeniería Ciencias Físicas y Matemáticas \\ Universidad Central del Ecuador \\ Ciudadela Universitaria Av. América, Quito, Ecuador \\ diegoquizanga@gmail.com \\ (3) Facultad de Ingeniería Civil y Ambiental \\ Escuela Politécnica Nacional \\ Av. Ladrón de Guevara E11-253, Quito, Ecuador \\ andrea.chicaiza@epn.edu.ec
}

\section{RESUMEN}

El presente estudio se enfoca en evaluar la peligrosidad sísmica local desde el punto de vista determinístico mediante la obtención de espectros de control para el valle de los Chillos, en especial para las parroquias Cotogchoa, Rumipamba, Sangolquí, San Pedro de Taboada y San Rafael, asociados a los tipos de suelos $C$ y D según la Norma Ecuatoriana de la Construcción (NEC-15).

Para ello, dos posibles escenarios sísmicos han sido planteados, el primero considerando el evento máximo generado por la ruptura del segmento Puengasí y el segundo debido a la falla Machachi, fallas que por la cercanía al área de estudio podrían causar efectos negativos en la población. Además se han obtenido los períodos de recurrencia, mediante los modelos del Terremoto Característico y Gutenberg-Richter Modificado.

Los modelos utilizados para encontrar los espectros de aceleración horizontal con $5 \%$ de amortiguamiento y para un nivel de confianza del $50 \%$ y $84 \%$ han sido los propuestos por Chiou \& Youngs (2013), Campbell \& Borzognia (2013) y Abrahamson, Silva y Kamai (2013), los cuales son aplicables para sismos corticales superficiales en regiones tectónicamente activas y pertenecientes al programa NGA-West2 del PEER.

Palabras Claves: Falla Machachi, Segmento de falla Puengasí, Período de recurrencia, Movimientos Fuertes, Espectros de Control. 


\begin{abstract}
This research focuses on evaluating local seismic hazard from a deterministic point of view by obtaining control spectra for Chillos valley especially for the parishes Cotogchoa, Rumipamba, Sangolquí, San Pedro de Taboada and San Rafael, which are associated with soil types $C$ and $D$ according to the "Norma Ecuatoriana de la Construcción 2015" (NEC-15).

For this purpose, two possible seismic scenarios have been set. The first is considering the maximum event generated by the rupture of fault segment Puengasí and the second is due to the Machachi fault, which because of proximity to the study area could cause negative effects on the population. In addition, recurrence periods have been obtained by the characteristic earthquake model and the modified Gutenberg-Richter.

The models used to find the horizontal acceleration spectra with $5 \%$ damping and a confidence level of $50 \%$ and $84 \%$ have been proposed by Chiou \& Youngs (2013), Campbell \& Borzognia (2013) and Abrahamson, Silva and Kamai (2013), which are applicable for shallow crustal earthquakes in active tectonic regions and belong to Pacific Earthquake Engineering Research Center's (PEER) NGA-West2 Program.
\end{abstract}

Keywords: Machachi Fault, Fault segment Puengasi, Recurrence Period, Strong motion, Control Spectra.

\title{
1. INTRODUCCIÓN
}

Ecuador es un país con alta peligrosidad sísmica por encontrarse ubicado en el "Cinturón de fuego del Pacífico", región donde ocurren al menos el $90 \%$ de toda la sismicidad mundial cada año. Además del fenómeno de subducción que se tiene frente a las costas ecuatorianas, el país se encuentra atravesado por un conjunto de fallas geológicas inactivas y activas, de las cuales esta última ha sido la causante de terremotos devastadores.

El valle de los Chillos al estar ubicado en una zona volcánica y sísmicamente activa, registra algunos acontecimientos sísmicos importantes uno de ellos fue el sismo del 9 de Agosto de 1938, conocido como el terremoto de los Chillos, cuya magnitud fue de Mw 5.8 y con epicentro en las cercanías del Tingo, Alangasí y Sangolquí, poblaciones donde muchas de las casas de adobe se destruyeron por completo y algunas casas de ladrillo resultaron con daños severos, ver figura 1.

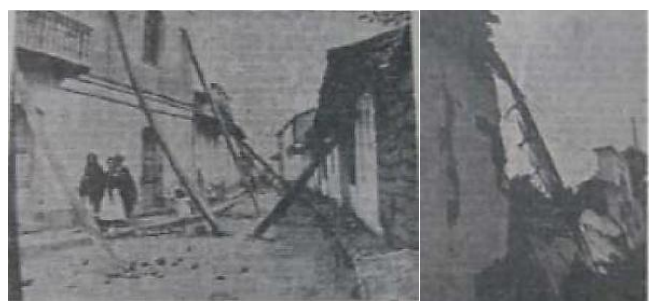

Figura 1 Daños ocasionados en el valle por efecto del sismo de 1938. Barona (2010). 
Para entender mejor la sismicidad producida en la zona de estudio debido a la incidencia de las fallas geológicas más cercanas, se recurre al programa ZONA_FUENTE elaborado por CEINCI-LAB.

La figura 2 muestra los epicentros de los sismos en la zona del Valle registrados entre los años 1541 y junio 2016, los mismos que están asociados a fallas locales con profundidad focal menor a $40 \mathrm{~km}$, mientras que la figura 3 presenta los epicentros de los sismos con profundidad focal mayor a $40 \mathrm{~km}$ para la misma zona.

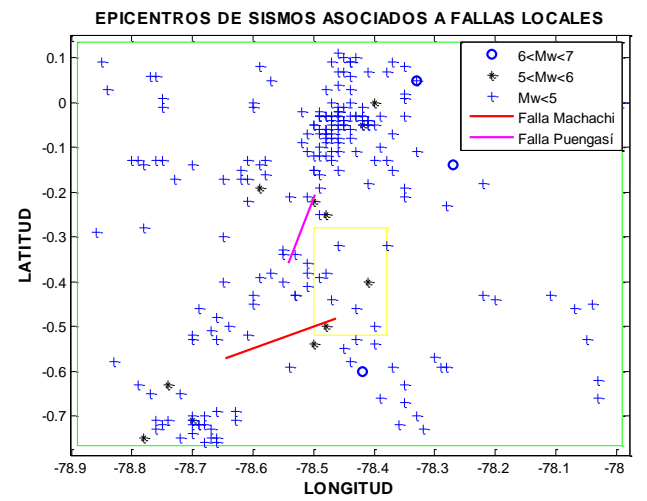

Figura 2 Epicentros de sismos asociados a fallas locales para la zona del Valle, profundidad focal menor a $40 \mathrm{~km}$.

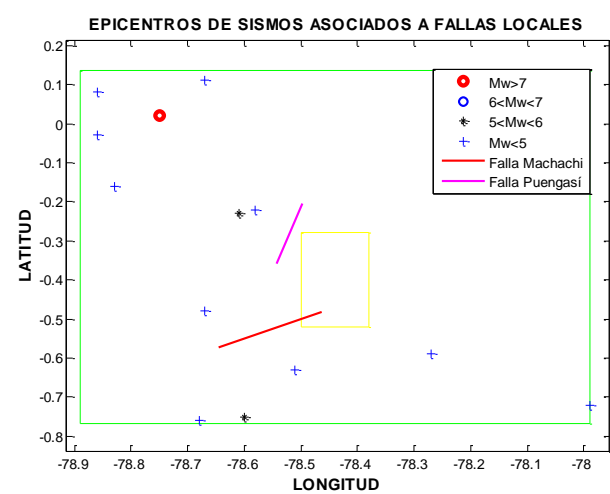

Figura 3 Epicentros de sismos asociados a fallas locales para la zona del valle, profundidad focal mayor a $40 \mathrm{~km}$.

La figura 2 muestra claramente que en la zona de estudio (recuadro color amarillo) existe el registro de dos sismos de magnitud Mw 5.9 en el año de 1929 y Mw 5.8 en 1938 (conocido como el Terremoto de los Chillos), en tanto que en la figura 3, se tiene el registro del sismo del año 1859 que tuvo una magnitud Mw 7.2 y causó mucho daño a la ciudad de Quito y sus alrededores, sin embargo en la zona de estudio no se muestra algún evento.

Al comparar las dos figuras, se puede evidenciar la gran influencia de sismos superficiales, que combinados con ciertos factores como la vulnerabilidad estructural pueden resultar muy peligrosos, es por eso su énfasis en este estudio. 


\section{MÁXIMA MAGNITUD GENERADA POR EL SEGMENTO DE FALLA PUENGASÍ}

El estudio de varios investigadores en el año 2012, permitió cuantificar la cinemática y la geometría del sistema de fallas de Quito en base a información geológica, geomorfológica e información sísmica (Quizanga, 2015).

Según Yepes (2014), el sistema de fallas de Quito es una falla del tipo inversa, en la que uno de los bloques (el de Quito) se levanta respecto del otro (los valles) en contra de la gravedad por fuerzas de compresión tectónica. Debido a esto, es por lo que Quito, a fuerza de sismos repetitivos en la historia geológica de su cuenca se levanta unos 400 metros sobre el nivel del Valle Interandino.

Así también en su publicación Alvarado et al. (2014), expresa la posibilidad de que dos escenarios sísmicos se produzcan, el primero cuando ocurra una ruptura simultánea de todos los segmentos que conforman la falla Quito, el cual podría generar magnitudes de $6.8(\mathrm{RA})$ y 7.1 (SRL) con un período de recurrencia posiblemente entre 195 y 235 años. Mientras que el segundo escenario, es la ruptura de los segmentos individuales del sistema de fallas de Quito, el mismo que es presentado en la tabla 1.

Tabla 1 Sistema de fallas Quito. Alvarado et al. (2014)

\begin{tabular}{|c|c|c|c|c|c|}
\hline $\begin{array}{c}\text { Sistema de Falla } \\
\text { Quito } \\
\text { Segmentos }\end{array}$ & $\begin{array}{c}\text { Área de } \\
\text { ruptura } \\
\left(\mathrm{km}^{2}\right)\end{array}$ & $\begin{array}{c}\text { Magnitud } \\
\text { (RA) }\end{array}$ & \begin{tabular}{|c|}
$\begin{array}{c}\text { Longitud } \\
\text { de ruptura } \\
(\mathrm{km})\end{array}$ \\
\end{tabular} & $\begin{array}{c}\text { Magnitud } \\
\text { (SRL) }\end{array}$ & $\begin{array}{c}\text { Período de } \\
\text { recurrencia } \\
\text { (años) }\end{array}$ \\
\hline Puengasí & 259 & 6.4 & 22 & 6.4 & 188 \\
\hline |lumbisí-La Bota & 176 & 6.2 & 15 & 6.2 & 138 \\
\hline Carcelén-El Inca & 82 & 5.9 & 7 & 5.7 & 105 \\
\hline Bellavista-Catequilla & 191 & 6.3 & 17.5 & 6.3 & 183 \\
\hline Tangahuilla & 108 & 6.0 & 12 & 6.0 & 115 \\
\hline
\end{tabular}

En la tabla 1 se puede observar que el segmento Puengasí podría generar un evento de magnitud Mw 6.4, produciendo la mayor magnitud respecto de los demás segmentos con un período de recurrencia de 188 años, obtenido mediante el modelo del Terremoto característico Wesnousky (1986).

Estas magnitudes máximas se hallaron en base a las ecuaciones de Leonard (2010) para fallas inversas, las cuales toman en cuenta la longitud de ruptura, ecuación (1) y área de ruptura, ecuación (2).

$$
\begin{gathered}
M w=1.52 \log (S R L)+4.4 \\
M w=1.0 \log (R A)+4
\end{gathered}
$$

Reemplazando la longitud y área de ruptura para el segmento Puengasí mostrado en la tabla 1, se tiene:

$$
\begin{gathered}
M w=1.52 \log (22)+4.4 \\
M w=6.4(S R L) \\
M w=1.0 \log (259)+4 \\
M w=6.4(R A)
\end{gathered}
$$




\section{MÁXIMA MAGNITUD GENERADA POR LA FALLA MACHACHI}

Soulas et al. (1991) identificaron esta falla por primera vez y se refirieron a ella como una parte del sistema de fallas Chingual - Pallatanga. Así mismo, en su publicación Chunga (2010) cita a esta falla como un nudo (zona de falla) sismogénico capaz de generar un sismo de magnitud mayor igual a 6 .

Egüez et al. (2003) definieron la geometría de esta falla de la siguiente manera:

- Longitud: $22.72 \mathrm{~km}$

- Rumbo promedio: $\mathrm{N} 60^{\circ} \mathrm{E} \pm 12^{\circ}$

- Buzamiento promedio: Desconocido.

- Sentido de movimiento: Dextral.

- Expresión Geomorfológica: Movimiento dextral a lo largo de la falla y asociados a fallas menores y secundarias normales y reversas.

- Velocidad de desplazamiento: Desconocido, probablemente $<1 \mathrm{~mm} / \mathrm{año}$.

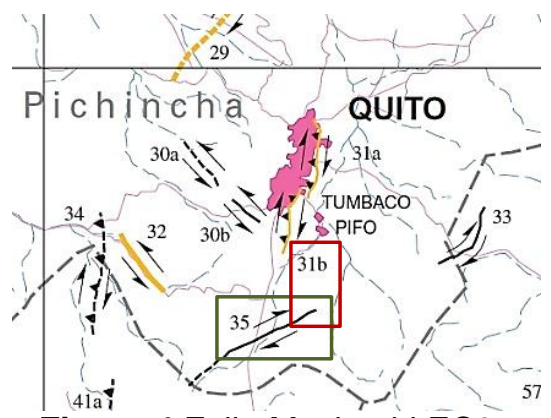

Figura 4 Falla Machachi EC35

Mapa de Fallas y Pliegues Cuaternarios de Ecuador y Regiones Oceánicas Adyacentes (2003).

En la figura 4, en el recuadro color verde se puede apreciar la falla Machachi (35) que es del tipo transcurrente dextral y su proximidad con la zona del valle (recuadro color rojizo).

Por otra parte, es necesario definir algunos parámetros de la falla entre ellos el ancho, el cual es encontrado mediante relaciones matemáticas expuestas en la tabla 5 de la publicación de Leonard (2010) y que está en función de longitud de falla como se puede observar en la ecuación (3).

$$
\log (W)=0.667 * \log (L)+1.18
$$

Reemplazando la longitud que tiene la falla Machachi, se obtiene un ancho de falla $W=12.18 \mathrm{~km}$ y un área de ruptura igual a $276.78 \mathrm{~km}^{2}$.

A continuación se determina la magnitud máxima que puede generar esta falla, utilizando igualmente las relaciones de Leonard (2010) para fallas transcurrentes (Strike Slip), las cuales relacionan la longitud superficial de ruptura $(\mathrm{SRL})$, ecuación (4) y el área de ruptura (RA), ecuación (5).

$$
M w=1.52 \log (S R L)+4.33
$$




$$
M w=1.0 \log (R A)+3.99
$$

Reemplazando la longitud de ruptura $22.7 \mathrm{~km}$ y $276.78 \mathrm{~km}^{2}$ de área de ruptura, se tiene un evento de magnitud Mw 6.4 (SRL) y Mw 6.4 (RA).

\section{PERÍODOS DE RECURRENCIA}

\subsection{Modelo del Terremoto Característico}

El período de recurrencia $(\mathrm{Tr})$ es el tiempo que transcurre para que se dé un sismo con las mismas características. Este modelo propuesto por Wesnousky (1986), supone que en la falla solamente se producen rupturas completas en todo el plano de falla, haciendo que la falla solo se rompa con sismos de la misma magnitud y que ocurre con periodicidad perfecta cada $\operatorname{Tr}$ años, imposibilitando de este modo que en la falla se produzcan sismos de magnitud inferior a la determinada (Aguiar, 2015; Rivas, 2014).

$$
\begin{gathered}
\dot{\mathrm{M} o}=\mu(\mathrm{u} . A) \\
T_{r}=\frac{M o}{\dot{\mathrm{M} o}}
\end{gathered}
$$

Donde Tr es el período de recurrencia; Mo momento sísmico; Mo tasa de acumulación del momento sísmico, $\mu$ módulo de deformación en rocas, ủ tasa de movimiento de la falla, $A$ área de ruptura.

\section{- Período de recurrencia hallado con el modelo del Terremoto Característico para el segmento Puengasí}

Alvarado et al. (2014) indican que el segmento Puengasí tiene un área de ruptura de $259 \mathrm{~km}^{2}$, con una tasa de movimiento de alrededor de $3 \mathrm{~mm} / \mathrm{año} \mathrm{y} \mathrm{un}$ módulo de deformación en roca de $3 \times 10^{10}$ Pascales.

Con estos datos y aplicando la ecuación 6 se ha obtenido una tasa de acumulación del momento sísmico de $2.36 \times 10^{16} \mathrm{~N}$.m/año.

Mientras que el momento sísmico que se liberaría en un terremoto de magnitud máxima ha sido despejado de la ecuación formulada en 1979 por Hanks y Kanamori:

$$
M_{w}=\frac{2}{3} \log (M o)-10.7
$$

Reemplazando la magnitud máxima Mw 6.4 en la ecuación 8, Mo es igual a $4.46 \times 10^{18} \mathrm{~N} . \mathrm{m}$, por lo tanto, el período de recurrencia obtenido fue 188 años, ratificándose de esta manera los valores calculados por Alvarado et al. (2014) que se muestra en la tabla 1 para el segmento Puengasí. 


\section{- Período de recurrencia hallado con el modelo del Terremoto Característico para la Falla Machachi}

De modo idéntico, se halla el período de recurrencia para la falla Machachi, obteniéndose un momento sísmico $\mathrm{Mo}=4.4668 \times 10^{18} \mathrm{~N}$.m y una tasa de acumulación del momento sísmico $\dot{M o}=8.3034 \times 10^{15} \mathrm{~N}$.m/año, la cual fue hallada con un módulo de deformación de las rocas durante el sismo igual a $3 \times 10^{10}$ Pascales, un desplazamiento de la falla de alrededor de $1 \mathrm{~mm} / \mathrm{año} \mathrm{y} \mathrm{un} \mathrm{área} \mathrm{de} \mathrm{ruptura} \mathrm{de} 276.78$ $\mathrm{km}^{2}$.

Calculándose de esta manera, un período de recurrencia de 538 años.

Tabla 2 Área de ruptura, magnitud máxima y período de recurrencia de la falla Machachi.

\begin{tabular}{|c|c|c|c|c|c|}
\hline Falla & $\begin{array}{c}\text { Área de } \\
\text { ruptura (RA) } \\
\mathbf{k m}^{2}\end{array}$ & $\begin{array}{c}\text { Longitud } \\
\text { Superficial } \\
\text { de ruptura } \\
\text { (RA) } \mathbf{k m}\end{array}$ & $\begin{array}{c}\text { Magnitud } \\
\text { (RA) }\end{array}$ & $\begin{array}{c}\text { Magnitud } \\
\text { (SRL) }\end{array}$ & $\begin{array}{c}\text { Período de } \\
\text { Recurrencia } \\
\text { (años) }\end{array}$ \\
\hline Machachi & 276.78 & 22.72 & 6.4 & 6.4 & 538 \\
\hline
\end{tabular}

En la tabla 2 se muestra los diferentes parámetros encontrados para la falla Machachi, entre ellos el área de ruptura, magnitud y período de recurrencia.

\subsection{Modelo de Gutenberg Richter Modificado}

Los modelos de recurrencia definen el potencial sísmico de una falla, relacionando parámetros como la frecuencia y el tamaño de sismos ocurridos en una fuente y tiempo determinado (Aguiar et al., 2016). Esta cuantificación ya fue formulada por Charles Francis Richter y Beno Gutenberg en 1944 y modificada por Consentino et al. (1977), integrando parámetros como; la tasa acumulada de sismos $[\dot{N}(m)]$ para diferentes magnitudes, la tasa acumulada de sismos de magnitud mayor o igual a una magnitud mínima establecida [ $\dot{N}_{M m i n}$ y la proporción de grandes versus pequeños sismos [b o $\beta$ ], mostrada en la siguiente ecuación:

$$
\dot{\mathrm{N}}(m)=\dot{\mathrm{N}}_{M \min } \cdot \frac{e^{-\beta(m)}-e^{-\beta(M \max )}}{\left(e^{-\beta(M \min )}-e^{-\beta(M \max )}\right)}
$$

Así mismo, la tasa de momento sísmico que se está acumulando en la falla M̀o, será liberada por medio de sismos de diferente magnitud (suponiendo un modelo de recurrencia tipo GR), las cuales variarán desde una magnitud $M_{\min }$, próxima a 0 , y la magnitud $\mathrm{M}_{\max }$ que vendrá definida por el tamaño del plano de falla. (Aguiar, 2015)

Se aplica la ecuación propuesta por Anderson (1979) que relaciona la tasa de momento acumulada con la tasa de momento que se libera.

$$
\dot{\mathrm{M}} o=\int_{M \min }^{M \max } \dot{\mathrm{n}}(m) M o(m) d m
$$

Donde la tasa de momento sísmico que se está acumulando en la falla, será igual a la tasa simple de sismos n் $(m)$ que se producen al año de magnitudes entre 
( $\left.\mathrm{M}_{\min }, \mathrm{M}_{\max }\right)$ por el momento sísmico que se libera en cada uno de esos sismos $M o$ (m). (Aguiar, 2015).

El momento sísmico que se libera en sismos de diferente magnitud, se obtiene por medio de la expresión propuesta por Hanks y Kanamori (1979). (Aguiar, 2015).

$$
M o(m)=e^{(c+d m)}
$$

Donde, $c=16.05 \ln (10)$ y $d=1.5 \ln (10)$.

Reemplazando las ecuaciones 9, 11 en la ecuación 10 se tiene:

$$
\begin{gathered}
\dot{\mathrm{M} o}=\int_{M \min }^{M \max } \dot{\mathrm{n}}(m) M o(m) d m=\int_{M \min }^{M \max } \dot{\mathrm{N} m i n} \cdot \beta\left[\frac{e^{-\beta m}}{\left(e^{-\beta(M \min )}-e^{-\beta(M \max )}\right.}\right] e^{c+d m} d m \\
\dot{\mathrm{M} o}=\frac{\dot{\mathrm{N}}_{M \min } \cdot \beta}{(d-\beta)\left(e^{-\beta M \min -e^{-\beta M \max }}\right)}\left[e^{-\beta M \max } M o(M \max )-e^{-\beta M \min } M o(M \min )\right]
\end{gathered}
$$

Despejando $\dot{\mathrm{N}}_{\mathrm{Mmin}}$, se tiene:

$$
\dot{\mathrm{N}}_{M \min }=\frac{\dot{\mathrm{M} o}(d-\beta)\left(e^{-\beta M \min }-e^{-\beta M \max }\right)}{\beta\left[e^{-\beta M \max } M o(\operatorname{Max})-e^{-\beta M \min } \operatorname{Mo}(\operatorname{Mmin})\right]}
$$

Donde Mo es la tasa de momento sísmico de la falla, Mo (Max) y Mo (Min) es el momento sísmico que se liberaría en los sismos de magnitud máxima y mínima respectivamente.

\section{- Período de Recurrencia hallado con el modelo de Gutenberg Richter Modificado}

Con un valor de $b=0.64$ obtenido para la zona $R \mid 3$ de la tabla 4.7 Parámetros de Sismicidad (Parra, 2016, p.102) y una tasa de deslizamiento para el segmento

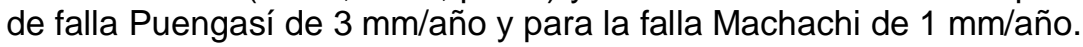

Además suponiendo que la falla puede generar sismos con magnitudes mayores a $M_{\min =4.0}$ hasta un $M_{\text {máx }}$ que es la magnitud máxima liberada en la falla obtenida con las relaciones de Leonard (2010) en función de la longitud o área del plano de ruptura. Se ha obtenido la tabla 3 que expresa el período de recurrencia calculado por medio de este modelo.

Tabla 3 Período de recurrencia hallados con el modelo Gutenberg y Richter Modificado para el segmento de falla Puengasí y la falla Machachi.

\begin{tabular}{|c|c|c|}
\hline $\begin{array}{c}\text { Rango de } \\
\text { magnitudes } \\
(\mathrm{Mw})\end{array}$ & \multicolumn{2}{|c|}{ Período de Recurrencia (Años) } \\
\cline { 2 - 3 }$\left[\begin{array}{c}\text { PUENGASI } \\
\text { [5.0-5.5) }\end{array}\right.$ & MACHACHI \\
\hline$[5.5-6.0)$ & $57-47$ & $65-132$ \\
\hline$[6-6.3]$ & $198-463$ & $161-409$ \\
\hline \multirow{2}{*}{ Mmax } & 999 & $555-1299$ \\
\cline { 2 - 3 } & $($ Mw6.4) & 2805 \\
\hline \multicolumn{2}{|c}{} \\
\hline
\end{tabular}




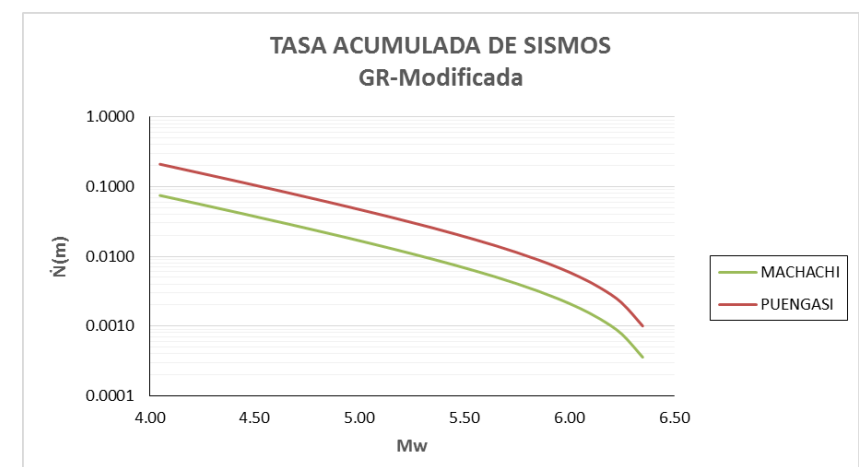

Figura 5 Tasa acumulada de sismos para el segmento de falla Puengasí y la falla Machachi Modelo GR-Modificada.

La figura 5 expresa la tasa acumulada de sismos $\dot{N}(\mathrm{~m})$ en función de la magnitud. La inversa de esta tasa acumulada expresa el período de recurrencia para cada magnitud asociada al segmento Puengasí (color rojo) y la falla Machachi (color verde).

\section{MODELOS DE MOVIMIENTO FUERTE}

Los modelos de movimiento fuerte cada vez han ido cobrando mayor interés en la ingeniería sísmica, pues la necesidad de conocer el movimiento esperado en un emplazamiento determinado debido a terremotos ocurridos, se ha vuelto indispensable en el diseño y construcción de las edificaciones y más en un país potencialmente sísmico como en el que habitamos.

Numerosas han sido las ecuaciones formuladas a nivel mundial para estimar la predicción del movimiento del suelo, pero entre ellas las más usadas y validadas mundialmente han sido las desarrolladas por el programa NGA-West del PEER (Pacific Earthquake Engineering Research Center's), en el cual se exponen los modelos propuestos por Abrahamson \& Silva (2008), Boore \& Atkinson (2008), Campbell \& Bozorgnia (2008), Chiou \& Youngs (2008) e Idriss (2008), es decir, un set de cinco modelos de atenuación del movimiento de suelo para regiones tectónicas activas, tales como California y varias partes del mundo.

Como parte de su actualización, el programa NGA-West2 permitió modelar de mejor manera ciertos parámetros utilizados en sus ecuaciones anteriores y ampliar la base de datos del programa original NGA-West, empleando alrededor de 21.336 registros para las tres componentes del movimiento del suelo provenientes de 600 eventos corticales superficiales registrados en todo el mundo.

Es así, que los modelos utilizados en la obtención de los espectros de control para este estudio, fueron los propuestos por Chiou \& Youngs (2013), Campbell \& Borzognia (2013) y Abrahamson et al. (2013), todos ellos pertenecientes al programa NGA-West2 del PEER y aplicables para sismos corticales superficiales en regiones tectónicamente activas.

Las ecuaciones de estos modelos toman en cuenta principalmente parámetros como el tipo de falla sea esta inversa, normal o transcurrente, el efecto 
del hanging-wall (bloque levantado), el efecto de la profundidad de sedimentos, el ángulo de buzamiento de la falla, la directividad y direccionalidad de la ruptura, la respuesta lineal y no lineal del suelo, atenuación anelástica y geométrica, desviaciones estándar entre y dentro del evento, entre otros.

Dicho lo anterior, es importante mencionar que esos parámetros han sido modelados utilizando técnicas de exploración de datos, tales como el análisis de los residuos, además que han sido desarrolladas a través de numerosas iteraciones para capturar las tendencias observadas en los datos de registros del movimiento del suelo (Campbell \& Borzognia, 2013) y que finalmente se vieron expresados mediante coeficientes en cada modelo.

\section{OBTENCIÓN DE ESPECTROS DE CONTROL}

Ahora bien para la determinación de los espectros de control se ha creado una malla de puntos con separación de 500 metros en los sentidos Norte-Sur y EsteOeste dentro de la zona definida, obteniéndose un total de 560 puntos, como se aprecia en la figura 6 .

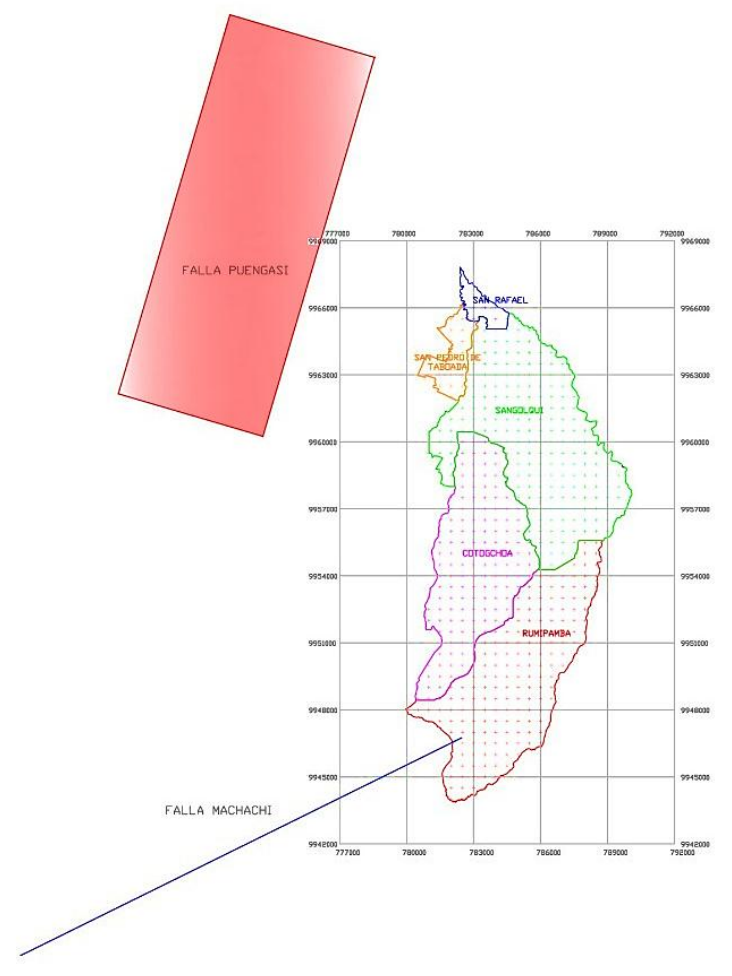

Figura 6 Plano de ruptura de las fallas geológicas Puengasí, Machachi y malla de puntos en coordenadas UTM.

La figura 6 muestra los 142 sitios seleccionados para la parroquia Cotogchoa, 173 para Rumipamba, 24 para San Pedro de Taboada, 12 para San Rafael y 209 para Sangolquí, dando un total de 560 sitios. Además se aprecia los planos de ruptura tanto inclinado y vertical del segmento Puengasí y la falla Machachi, respectivamente. 
Pese a que la zona no cuenta con estudios que permitan determinar el parámetro $\mathrm{V}_{\mathrm{s} 30}$ para cada uno de los 560 sitios establecidos, se recurre a varios estudios de suelos realizados en Quito, donde Quizanga (2015) ha encontrado que un valor representativo de la velocidad de onda de corte $V_{s 30}$ es $460 \mathrm{~m} / \mathrm{s}$ para suelos tipo $C$ y $300 \mathrm{~m} / \mathrm{s}$ para suelos tipo D, acorde a la clasificación de suelos de la Norma Ecuatoriana de la Construcción 2015 (NEC-15).

Por consiguiente, de la figura 7 a la 11 se presentan los espectros que registran las mayores aceleraciones dentro de cada parroquia y para cada modelo Chiou \& Youngs (2013) [CY13], Campbell \& Borzognia (2013) [CB13] y Abrahamson et al. (2013) [ASK13] y que además están asociados al segmento Puengasí y la falla Machachi y a los tipos de suelos $C$ y D. Conjuntamente se ha colocado el espectro promedio entre los tres modelos indicados y el espectro de diseño de la NEC15.

El nivel de confianza que se espera en la obtención de estos espectros es del $50 \%$ cuando no se considera la desviación estándar (línea continua) y $84 \%$ cuando se la incluye una vez (línea discontinua).

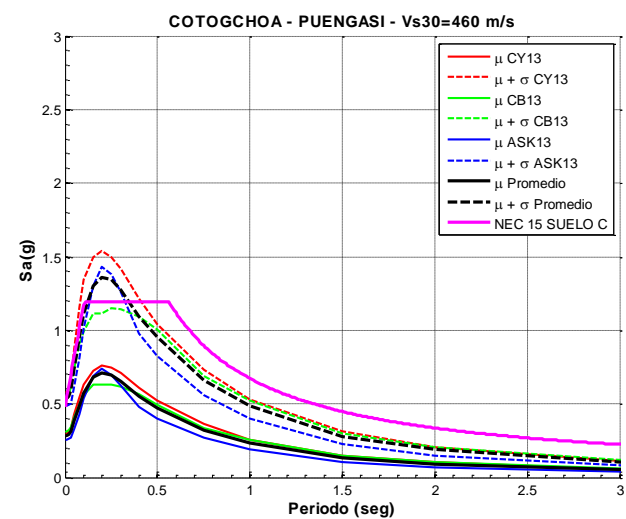

a)

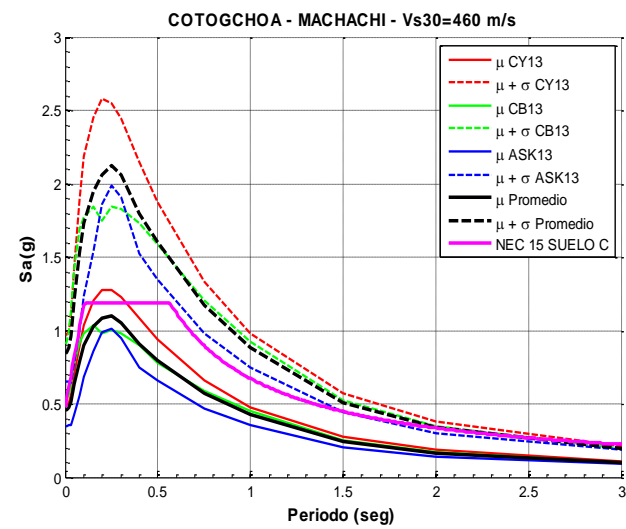

c)

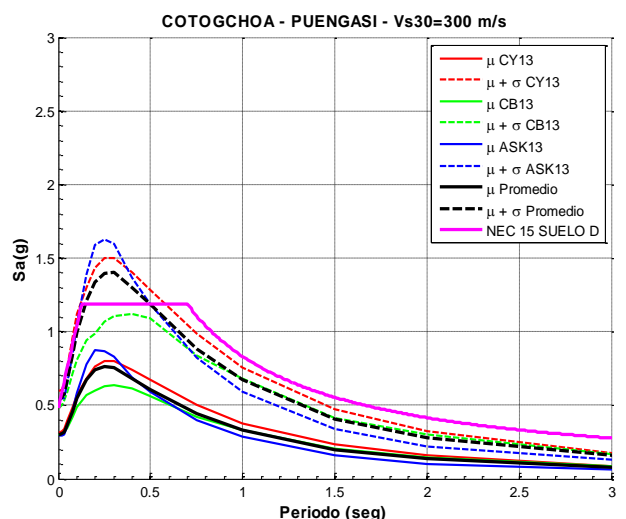

b)

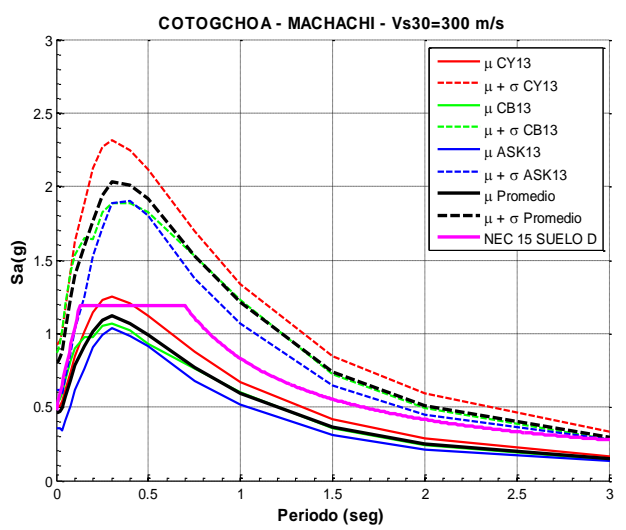

d)

Figura 7 Espectros críticos para Cotogchoa: a) Espectro asociado al segmento Puengasí en suelo tipo $\mathrm{C}$ b) Espectro asociado al segmento Puengasí en suelo tipo $D$, c) Espectro asociado a la falla Machachi en suelo tipo C, d) Espectro asociado a la falla Machachi en suelo tipo D. 


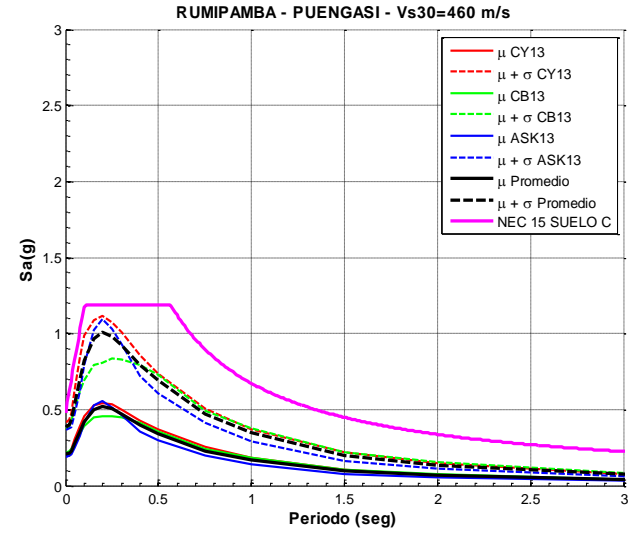

a)

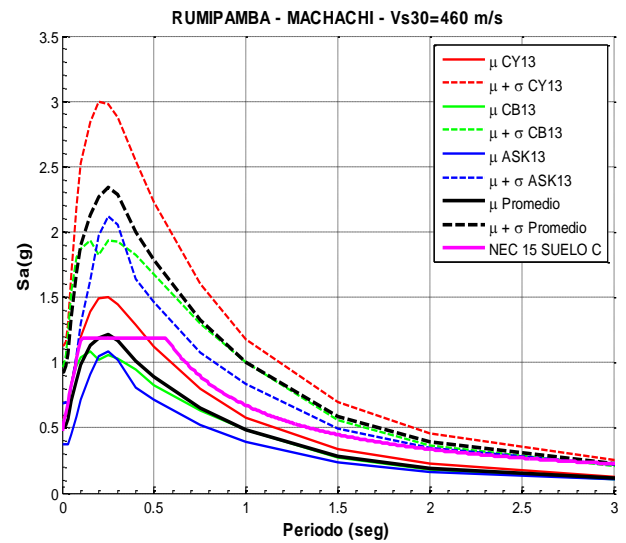

c)

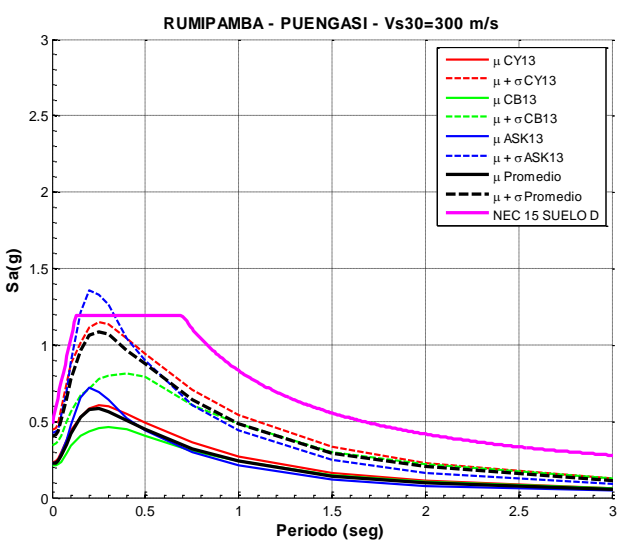

b)

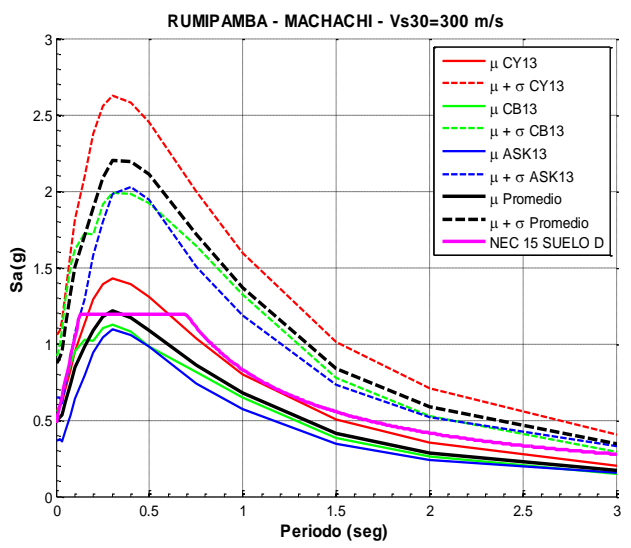

d)

Figura 8 Espectros críticos para Rumipamba: a) Espectro asociado al segmento Puengasí en suelo tipo $\mathrm{C}$ b) Espectro asociado al segmento Puengasí en suelo tipo $D$, c) Espectro asociado a la falla Machachi en suelo tipo C, d) Espectro asociado a la falla Machachi en suelo tipo D.

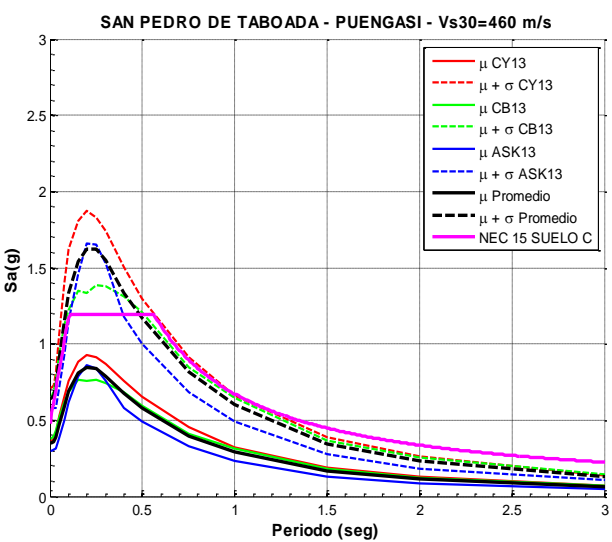

a)

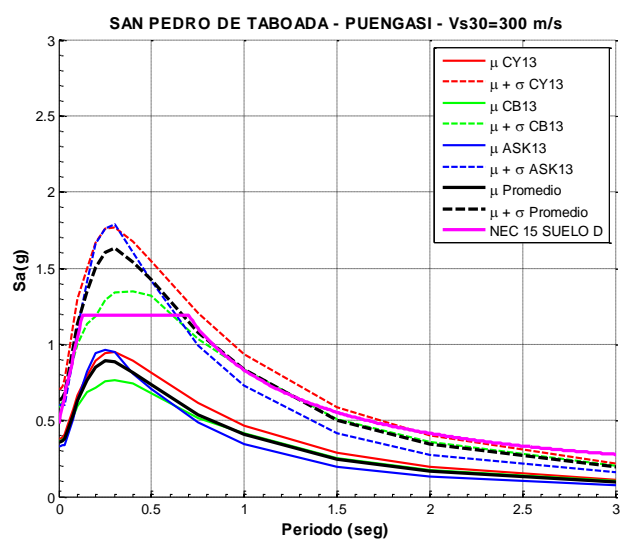

b) 


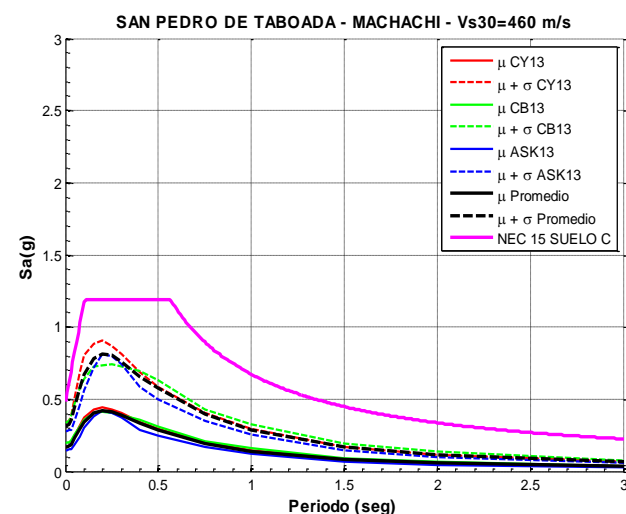

c)

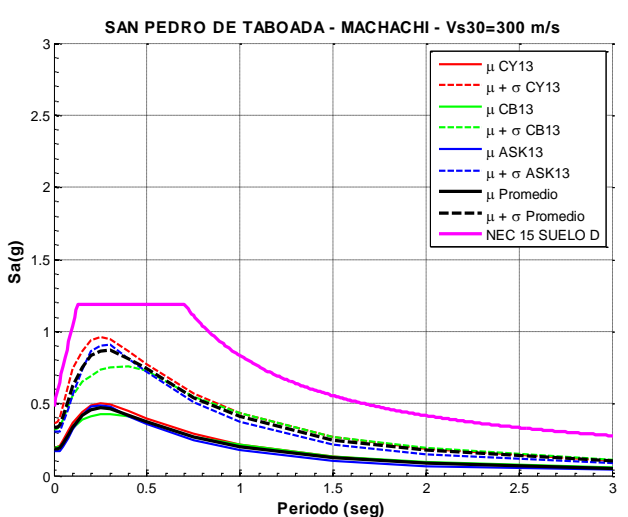

d)

Figura 9 Espectros críticos para San Pedro de Taboada: a) Espectro asociado al segmento Puengasí en suelo tipo $\mathrm{C}$ b) Espectro asociado al segmento Puengasí en suelo tipo D, c) Espectro asociado a la falla Machachi en suelo tipo C, d) Espectro asociado a la falla Machachi en suelo tipo $D$.

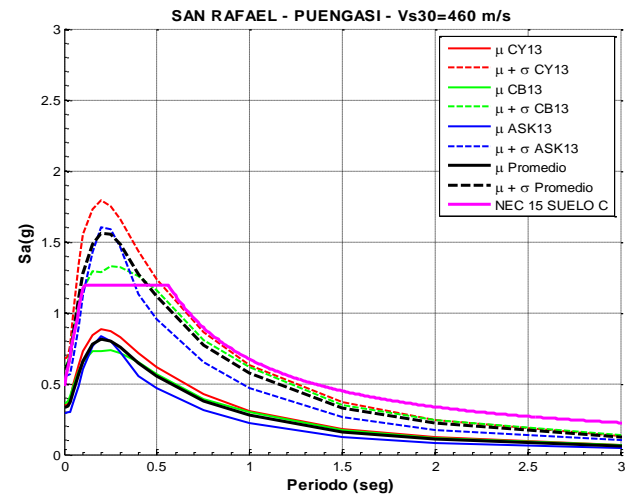

a)

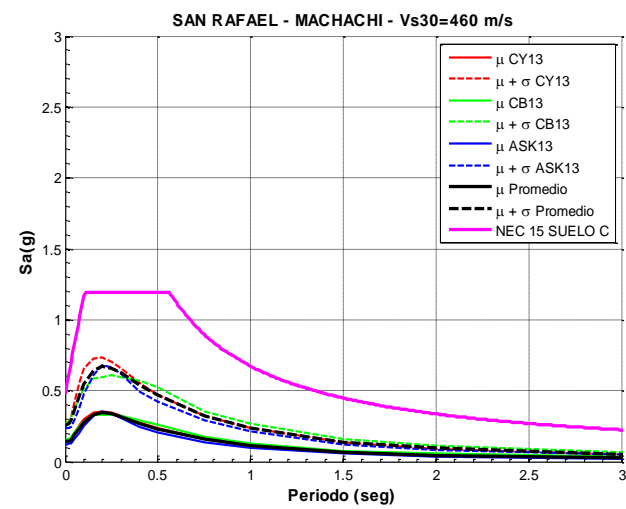

c)

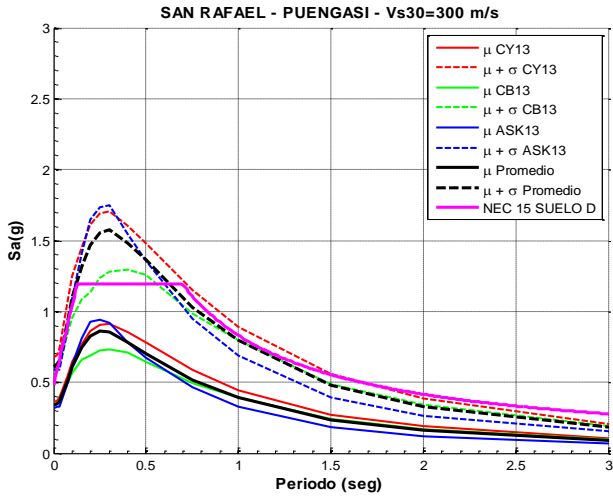

b)

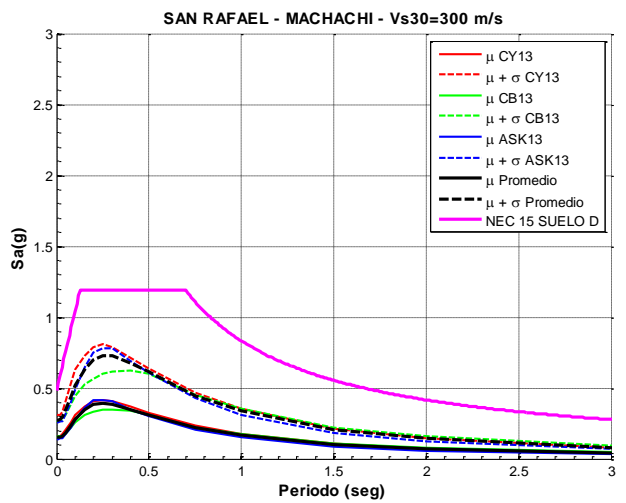

d)

Figura 10 Espectros críticos para San Rafael: a) Espectro asociado al segmento Puengasí en suelo tipo $\mathrm{C}$ b) Espectro asociado al segmento Puengasí en suelo tipo D, c) Espectro asociado a la falla Machachi en suelo tipo C, d) Espectro asociado a la falla Machachi en suelo tipo D. 


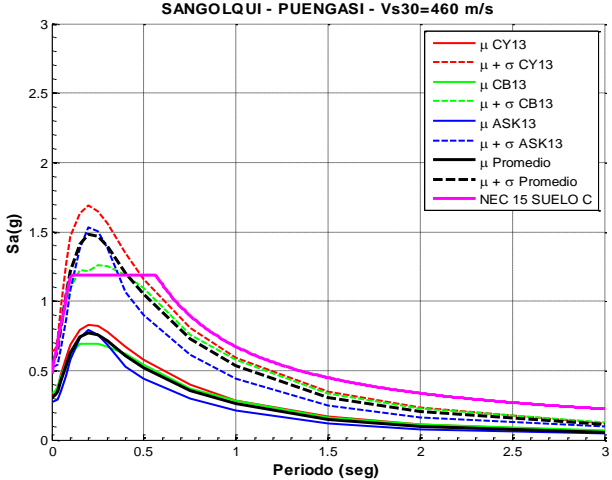

a)

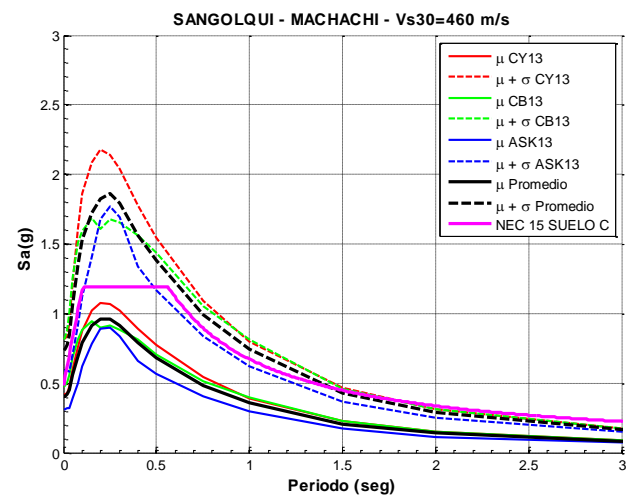

c)

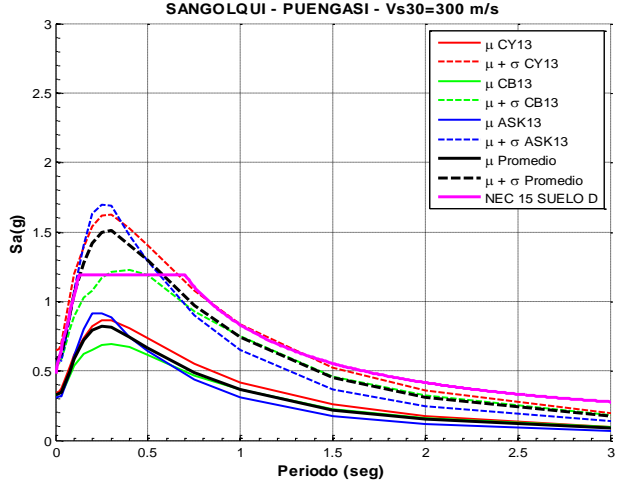

b)

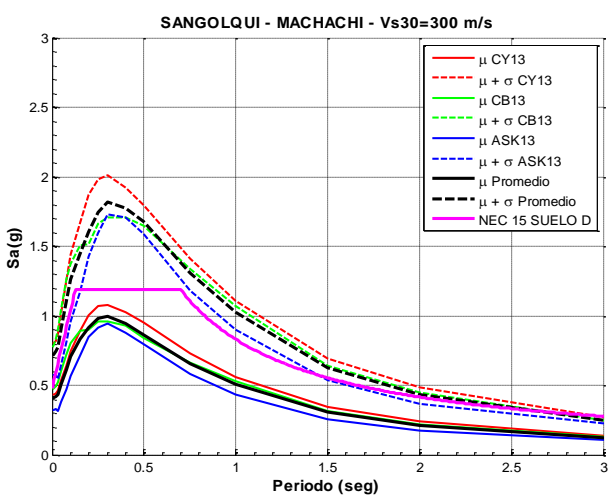

d)

Figura 11 Espectros críticos para Sangolquí: a) Espectro asociado al segmento Puengasí en suelo tipo $C$ b) Espectro asociado al segmento Puengasí en suelo tipo D, c) Espectro asociado a la falla Machachi en suelo tipo C, d) Espectro asociado a la falla Machachi en suelo tipo D.

A continuación las figuras 12 y 13 muestran los espectros que se han obtenido en base al promedio de las aceleraciones espectrales de los tres modelos CY13, CB13 y ASK13 para cada parroquia, asociada a una falla y un tipo de suelo.

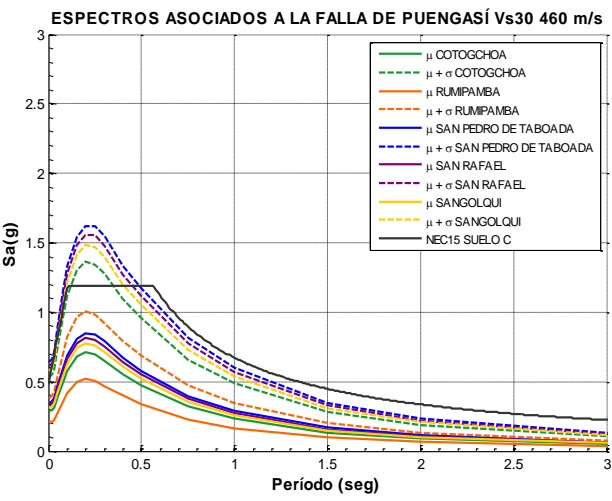

a)

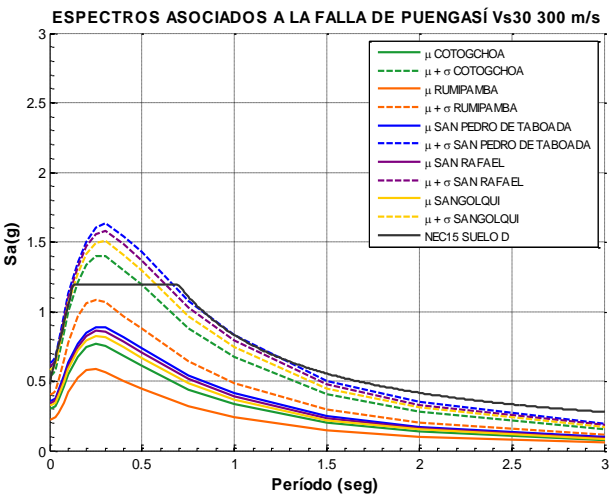

b)

Figura 12 Espectros promedios de cada parroquia respecto al segmento Puengasí: a) asociado a suelo tipo $C$, b) asociado a suelo tipo $D$. 


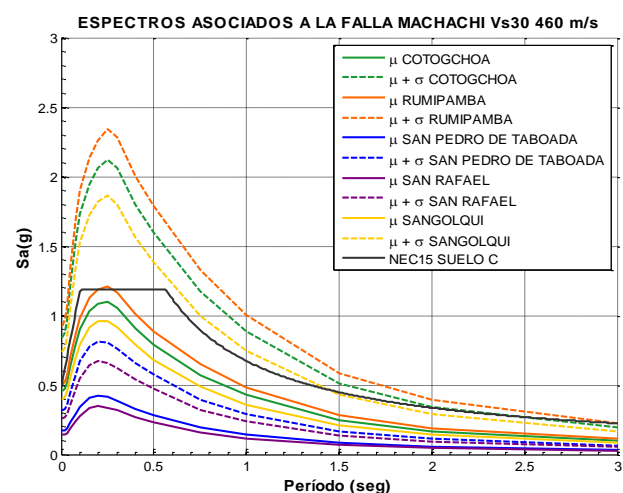

a)

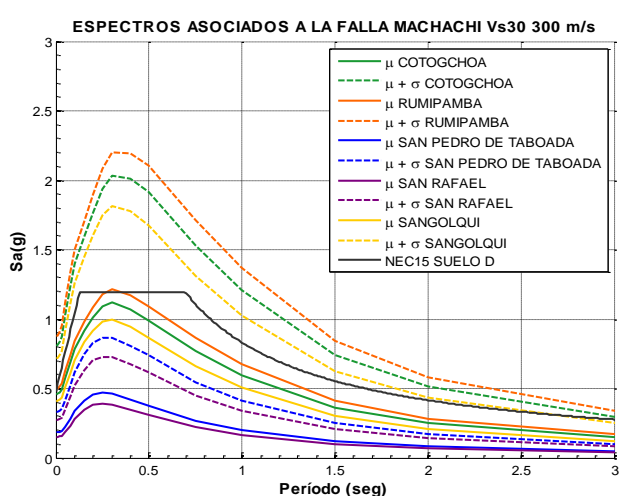

b)

Figura 13 Espectros promedios de cada parroquia respecto a la falla Machachi: a) asociado a suelo tipo $\mathrm{C}, \mathbf{b})$ asociado a suelo tipo $\mathrm{D}$.

Acorde a las figuras 12 y 13 , se puede acotar que la falla Machachi genera mayores aceleraciones en las parroquias de Cotogchoa, Rumipamba y Sangolquí, siendo estas entre $1.2(\mathrm{~g})$ a $2.3(\mathrm{~g})$ dependiendo el nivel de confianza que se quiera evaluar ( $50 \%$ y $84 \%$ respectivamente) mientras que debido al segmento de falla Puengasí, las parroquias San Rafael, San Pedro de Taboada y también Sangolquí registran aceleraciones entre $0.9(\mathrm{~g})$ a $1.6(\mathrm{~g})$.

Por último, en la tabla 4 se muestra los valores más críticos de PGA obtenidos para cada parroquia debido a los eventos originados por el segmento Puengasí y la falla Machachi evaluados en los dos tipos de suelos y para un nivel de confianza del $84 \%$, conjuntamente se expone el PGA hallado mediante la norma NEC-15. De esta manera se determina un factor de 1.5 para encontrar el sismo máximo considerado a partir del sismo de diseño la NEC-15 para esta zona.

Tabla 4 Factor para encontrar el sismo máximo considerado a partir del sismo de diseño de la NEC-15.

\begin{tabular}{|c|c|c|c|c|c|c|c|}
\hline \multicolumn{5}{|c|}{ Modelos de Movimiento Fuerte } & \multirow{2}{*}{$\begin{array}{c}\text { Factor } \\
\text { Diseño } \\
\text { (Promedio/ } \\
\text { NEC-15 }\end{array}$} & $\begin{array}{c}\text { Diseño } \\
\text { NEC-15) }\end{array}$ \\
\cline { 1 - 5 } Rumipamba & Cotogchoa & $\begin{array}{c}\text { San Pedro de } \\
\text { Taboada }\end{array}$ & San Rafael & Sangolquí & Promedio & & \\
\hline 0.90 & 0.82 & 0.63 & 0.61 & 0.65 & 0.72 & 0.48 & $\mathbf{1 . 5 0}$ \\
\hline
\end{tabular}

\section{COMENTARIOS Y CONCLUSIONES}

Los sismos corticales de mayor magnitud que se podrían esperar en el valle, producto de las fallas geológicas Puengasí y Machachi corresponde a una magnitud máxima de $\mathrm{Mw} 6.4$ con un periodo de recurrencia de 188 y 538 años respectivamente.

Los modelos que registran similares aceleraciones espectrales debido al evento generado por el segmento Puengasí (falla inversa) y para los dos niveles de confianza $50 \%$ y $84 \%$ son Chiou \& Youngs (2013) y Abrahamson et al. (2013), mientras que debido a la falla Machachi (falla transcurrente) son los modelos Abrahamson et al. (2013) y Campbell \& Borzognia (2013). Por lo que se debe tomar 
a consideración estos modelos en la determinación de espectros para la zona del valle.

Las parroquias que registran las mayores aceleraciones espectrales debido al evento sísmico generado por el segmento Puengasí y tomando en cuenta un nivel de confianza del $84 \%$ son San Pedro de Taboada, San Rafael y Sangolquí, con aceleraciones espectrales cercanas a $1.6 \mathrm{~g}$ ) y un PGA de $0.6 \mathrm{~g}$ (g), teniéndose un incremento del $30 \%$ respecto a las obtenidas mediante la norma.

Entre tanto que las parroquias que registran las mayores aceleraciones espectrales debido al evento sísmico generado por la falla Machachi y tomando en cuenta un nivel de confianza $84 \%$ son Cotogchoa, Rumipamba y Sangolquí, con aceleraciones espectrales cercanas a $2.3(\mathrm{~g})$ y un PGA de $0.8(\mathrm{~g})$, teniéndose un incremento del $70 \%$ respecto a las obtenidas mediante la norma.

De los dos posibles escenarios sísmicos planteados producto de las dos fallas cercanas a la zona de estudio, el caso más crítico será cuando se produzca el evento debido a la falla Machachi ya que parroquias como Cotogchoa y Rumipamba, soportarían aceleraciones mayores al doble de la gravedad, aunque también se debe tener en cuenta el período de recurrencia que la misma tendrá, pese a esto de cierta forma es preocupante porque el espectro obtenido por la NEC 15 no cubre estos valores.

Los períodos donde se registran las mayores aceleraciones para cada parroquia está en el rango de 0.2 a 0.3 segundos, por lo que, las edificaciones que están entre 2 a 4 pisos aproximadamente serán las que reciban mayores fuerzas sísmicas producto de los eventos originados por estas fallas, cabe recalcar que estas construcciones son muy típicas en la zona de estudio.

Se sugiere verificar el desempeño sísmico esperado de las estructuras del lugar con el espectro del sismo máximo considerado que es el que se expone en esta investigación para un nivel de confianza del $84 \%$, el cual ha sido determinado para esta zona como 1.5 veces más que el espectro del sismo de diseño obtenido por la NEC-15.

\section{REFERENCIAS}

1. Abrahamson N., Silva W., Kamai R., (2013), Update of the AS08 GroundMotion Prediction Equations Based on the NGA-West2 Data Set, Pacific Earthquake Engineering Research Center, PEER, $143 \mathrm{p}$.

2. Aguiar R., (2015), Espectros de Control para la ciudad de Quito, Centro Internacional de Métodos Numéricos, CIMNE, Monografías de Ingeniería Sísmica, IS-72, Universidad Politécnica de Cataluña, 104 p.

3. Aguiar R., Rivas A, Caiza P y Quizanga D, (2016), "Control Spectra for Quito", Journal Nat. Hazards Earth Syst. Sci. Discuss., doi: 10.5194/nhess2016-33, 19 p. Article online.

4. Alvarado A., Audin L., Nocquet M., Lagreulet S., Segovia M., Font Y., Lamarque G., Yepes H., Mothes P., Rolandone F., Jarrín P., and Quidelleur X., (2014), "Active tectonics in Quito, Ecuador, assessed by geomorpholigical studies, GPS data, and crustal seismicity", Tectonics, AGUPLICATIONS, 17 p., Article online. 
5. Barona D., (2010), Vulnerabilidad sísmica del Centro Histórico de Sangolquí, Tesis de Grado, Universidad de las Fuerzas Armadas, ESPE, $143 \mathrm{p}$.

6. Campbell K., Bozorgnia Y., (2013), NGA-West2 Campbell-Bozorgnia ground motion model for the horizontal components of PGA, PGV and 5\%Damped elastic Pseudo-Acceleration response spectra for periods ranging for 0.01 to $10 \mathrm{sec}$, Pacific Earthquake Engineering Research Center, PEER, $75 \mathrm{p}$.

7. Chiou B. y Youngs R., (2013), Update of the Chiou and Youngs NGA Ground Motion Model for Average Horizontal Component of Peak Ground Motion and Response Spectra, Pacific Earthquake Engineering Research Center, PEER, $78 \mathrm{p}$.

8. Chunga K., Michetti A., Gorshkov A., Panza G., Soloviev A. y Martillo C., (2010), "Aplicación del método de zonación morfo-estructural para identificar nudos sismogénicos en la región costera y cadenas montañosas de los andes septentrionales del Ecuador", Acta Oceanográfica del Pacífico, 16 (1), 1-144.

9. Egüez A., Alvarado A., Yepes H., Machette M., Costa C. y Dart R., (2003), Database and map of Quaternary faults and Fonds of Ecuador and its offshore regions, U.S. Geological Survey, Open-File Report 03-289, 77 p.

10. Leonard M., (2010), "Earthquake fault scaling: Self consistent relating of rupture length width, average displacement, and moment release", Bull. Seismol. Soc. Am, 100 (5A), 1971-1988.

11. NEC, (2014), Norma Ecuatoriana de la Construcción, Ministerio de Desarrollo Urbano y Vivienda, MIDUVI.

12. Parra H., (2016), Desarrollos metodológicos y aplicaciones hacia el cálculo de la peligrosidad sísmica en el ecuador continental y estudio de riesgo sísmico en la ciudad de Quito, Tesis Doctoral, Universidad Politécnica de Madrid, $242 \mathrm{p}$.

13. Quizanga D.,(2015), Espectros específicos para la ciudad de Quito en función de fallas ciegas inversas, Tesis de Maestría, Escuela Politécnica Nacional, 170 p., Quito.

14. Rivas A., (2014), Contribución metodológica para incorporar fallas activas en la modelización de la fuente dirigida a estimaciones de peligrosidad sísmica. Aplicación al sur de España, Tesis Doctoral, Universidad Politécnica de Madrid, $235 \mathrm{p}$.

15. Soulas J.P., Egüez A., Yepes H. y Pérez H., (1991), "Tectónica activa y riesgo sísmico en los Andes Ecuatorianos y el extremo sur de Colombia", Bol. Geol. Ecuat., 2 (1), 3-11, Quito.

16. Yepes H., (2014), Breve reseña de los sismos provenientes de la falla geológica de Quito que han afectado a la Capital, Instituto Geofísico Escuela Politécnica Nacional, Quito.

https://www.igepn.edu.ec 